\section{Increased systemic oxidative stress in patients with keratoconus}

I Toprak 1, V Kucukatay², C Yildirim ${ }^{3,4}$,

E Kilic-Toprak ${ }^{2}$ and O Kilic-Erkek ${ }^{2}$

\begin{abstract}
Purpose To establish the effect of systemic oxidative stress on the pathogenesis of keratoconus by measuring serum total oxidant status (TOS) and total antioxidant status (TAS) in patients with keratoconus. Methods Twenty-five patients with keratoconus (keratoconus group) and 25 age-sex-matched healthy subjects (control group) were enrolled in the study. Exclusion criteria were smoking habit, history of any other corneal pathology, systemic disease or inflammation, and current antioxidant or anti-inflammatory therapies. All participants underwent a detailed ophthalmological examination and corneal topography. Serum samples were obtained from all participants. Oxidative stress markers (TAS and TOS) were measured using a commercial kit and oxidative stress index (OSI) was calculated. Results The study comprised 25 patients with keratoconus (mean age of $26.4 \pm 1.7$ years) and 25 healthy control subjects (mean age of $26.6 \pm 1.7$ years) $(P>0.05)$. The serum TOS and OSI values were significantly higher in patients with keratoconus compared with those of the controls $(P=0.036$ and 0.037 , respectively). However, serum TAS did not show significant difference between the keratoconus and control groups $(P=0.497)$. Conclusions The higher levels of serum oxidant status and OSI in patients with keratoconus suggest that systemic oxidative stress might be involved in the pathogenesis of keratoconus.
\end{abstract}

Eye (2014) 28, 285-289; doi:10.1038/eye.2013.262; published online 6 December 2013

Keywords: antioxidant status; keratoconus; oxidant status; oxidative stress

\section{Introduction}

Keratoconus is an ectatic corneal disorder characterized by progressive stromal thinning and irregular astigmatism that occurs in young adults ${ }^{1,2}$ Major clinical findings are focal scarring, breaks in Bowman's layer, and iron deposits within the epithelium. ${ }^{2}$ Although keratoconus is defined as a non-inflammatory corneal disease and the molecular pathophysiology of keratoconus continues to be elucidated, recent studies suggest that a combination of genetic and environmental risk factors, such as eye rubbing, atopy, inflammation, and oxidative stress, play a role in the pathogenesis. ${ }^{1-3}$

Cornea as a transparent tissue absorbs most of the ultraviolet (UV) light entering the eye and UV is an important environmental stress factor that creates cytotoxic free radicals. ${ }^{4}$ It could be suggested that the cornea is exposed to free radicals that lead to cellular damage by reacting with proteins, DNA, and membrane

phospholipids. In the healthy cornea, a number of defensive mechanisms such as superoxide dismutase, catalase, glutathione peroxidase, and glutathione reductase are present to eliminate oxidative byproducts. ${ }^{4,5}$ However, keratoconic corneas have accumulation of cytotoxic byproducts (such as superoxides, hydrogen peroxide, and hydroxyl radicals) and abnormalities in antioxidant defense mechanisms. ${ }^{6-8}$ More recently, corneal thinning in the keratoconus has been found to be associated with increase in tissue proteinase activities and decrease in proteinase inhibitor levels induced by oxidant products. ${ }^{9}$

Free radicals are produced in metabolic and physiological processes, and harmful oxidative reactions may occur in organisms. ${ }^{10}$ Organisms are protected against oxidative stress via enzymatic and non-enzymatic antioxidative mechanisms. ${ }^{10}$ Under normal conditions, a delicate balance exists between rates of free radical formation and their removal by antioxidant enzymes and molecules.

Oxidative stress has been implicated in the pathophysiology of many systemic and ocular
'Department of Ophthalmology, Servergazi State Hospital, Denizli, Turkey

${ }^{2}$ Department of Physiology, Faculty of Medicine, Pamukkale University, Denizli, Turkey

${ }^{3}$ Department of Ophthalmology, Faculty of Medicine, Pamukkale University, Denizli, Turkey

${ }^{4}$ Private Ege Akademi Eye Hospital, Denizli, Turkey

Correspondence:

I Toprak, Department of Ophthalmology, Servergazi State Hospital, Bereketli Beldesi, Denizli 20070, Turkey.

Tel: +90 5054953791 ; Fax: +90 2583613101 .

E-mail: ibrahimt@

doctor.com

Received: 15 February 2013 Accepted in revised form: 11 October 2013 Published online: 6 December 2013

The abstract of this study was presented as poster presentation for the Congress of the European Society of Ophthalmology (SOE) 2013 (8-11 June 2013, Copenhagen, Denmark). 
diseases such as glaucoma, retinopathy of prematurity, macular degeneration, and uveitis. ${ }^{11}$ Although previous ex vivo studies have reported oxidative damage in keratoconic corneas, systemic oxidant and antioxidant status were not investigated in patients with keratoconus previously. ${ }^{4-6}$ The oxidative status of biological samples is regarded as an indicator of oxidative stress and the measurement of total oxidant status (TOS) and total antioxidant status (TAS) are the most common procedures to test for prediction of oxidative stress. ${ }^{10,11}$ Hence, the aim of the present study was to establish the role of systemic oxidative stress in the pathogenesis of keratoconus. For this purpose, we used the total oxidant and antioxidant parameters instead of individual compounds to assess the cumulative capacity of oxidant and antioxidant components.

\section{Materials and methods}

\section{Study population}

The study was performed in adherence to the tenets of the Declaration of Helsinki and the local ethics committee approved the methodology. Written informed consent was obtained from all participants. Twenty-five patients with keratoconus (keratoconus group) and 25 age-sex-matched healthy subjects (control group) with normal ophthalmological examination were enrolled in this study.

Inclusion criteria for the keratoconus group were corneal topography pattern consistent with keratoconus, inferior-superior ratio on topographic map $>1.5$, and at least 18 years of age. Subjects with any of following conditions were excluded from the study: smoking habit, history of any other corneal pathology or surgery, presence of systemic inflammation or systemic disease (diabetes, uncontrolled hypertension, renal, or hepatic dysfunction), and current anti-inflammatory or antioxidant therapies.

All participants underwent a complete ophthalmic examination including corrected visual acuity measurement (Snellen charts), slit-lamp biomicroscopy examination, applanation tonometry, and dilated funduscopic examination with non-contact $+90 \mathrm{D}$ lens.

Corneal topography (Oculus Pentacam, Oculus Optikgerate $\mathrm{GmbH}$, Wetzlar, Germany) was conducted with undilated pupils under scotopic conditions by a single experienced technician. Scans were taken in automatic mode with proper quality. In bilateral cases, eyes with more advanced stage of keratoconus (eyes with a steeper maximum keratometry) were selected for statistical analysis. Eyes were divided into groups according to the maximum keratometry as follows: mild (maximum $K \leq 47 \mathrm{D}$ ), moderate (maximum $K$ between 47-52 D), and severe (maximum $K \geq 52 \mathrm{D}$ ) keratoconus.

\section{Blood sampling}

Five milliliters of peripheral venous blood was obtained from all participants after overnight fasting in the morning. The blood samples were collected into plain red top blood collection tubes (without any solution) and centrifuged at 5000 r.p.m. for $10 \mathrm{~min}$. The serum layer was separated and stored at $-80^{\circ} \mathrm{C}$ until needed for analysis.

\section{TOS measurement}

The TOS of serum was determined using an automated measurement method developed by Erel. ${ }^{12}$ In this method; oxidants present in the sample oxidize the ferrous ion-o-dianisidine complex to ferric ion. The oxidation reaction is enhanced by the glycerol molecules present in the reaction medium. The ferric ion produced a colored complex with xylenol orange in acidic solution. The color intensity, which is measured spectrophotometrically, is proportional to the total amount of oxidant molecules present in the sample. The assay is calibrated with hydrogen peroxide, and the results are expressed in terms of micromolar hydrogen peroxide equivalents per liter $\left(\mu \mathrm{mol} \mathrm{H}_{2} \mathrm{O}_{2}\right.$ equivalent/1). ${ }^{12}$

\section{TAS measurement}

The TAS of the serum was determined using a novel automated measurement method developed by Erel. ${ }^{10}$ In this method, hydroxyl radical, which is the most potent biological radical, is produced by the Fenton reaction and reacts with the colorless substrate $\mathrm{O}$-dianisidine to produce the dianisyl radical, which is bright yellowishbrown in color. Upon the addition of a serum sample, the oxidative reactions initiated by the hydroxyl radicals present in the reaction mix are suppressed by the antioxidant components of the serum, preventing the color change and thereby providing an effective measure of the total antioxidant capacity of the serum. ${ }^{10}$ The results were expressed as mmol Trolox equivalent/1.

\section{Oxidative stress index}

The percentage ratio of the total peroxide level to the TAS was used as the oxidative stress index (OSI). ${ }^{13}$ The OSI was calculated according to the following formula: ${ }^{13}$

OSI (arbitrary unit) $=$ TOS $\left(\mu \mathrm{mol} \mathrm{H}_{2} \mathrm{O}_{2} \mathrm{Eq} / \mathrm{l}\right) / \mathrm{TAS}$ $(\mu \mathrm{mol}$ Trolox Eq/1) $\times 100$ 


\section{Statistical analysis}

Sample size was determined at $92 \%$ power and 0.05 significance (95\% confidence interval) level from previous similar literature and was found to be 19 subjects per group using a software (PASS version 11.0.1, NSCC, LLC, Park City, UT, USA). Statistical analysis was performed with the Statistical Package for Social Sciences software version 16.0 (SPSS Inc, Chicago, IL, USA). The values were expressed as mean \pm standard error (SE). Qualitative variables were analyzed using the $\chi^{2}$ test. Comparison of the quantitative data (TAS, TOS, and OSI values) between two groups was performed by Mann-Whitney $U$ test. Kruskal-Wallis test was used to compare differences among keratoconus severity groups in terms of oxidative stress markers (TAS, TOS, and OSI values). Spearman correlation coefficients were used to analyze the association between maximum keratometry and oxidative stress markers (TAS, TOS, and OSI values). A $P$-value less than 0.05 was considered statistically significant at $95 \%$ confidence interval.

\section{Results}

Twenty-five patients with keratoconus (keratoconus group) and 25 age-sex matched healthy subjects (control group) were included in the study. The keratoconus group consisted of $12(48 \%)$ male and $13(52 \%)$ female (mean age $26.4 \pm 1.7$ years) and the control group comprised $11(44 \%)$ male and $14(56 \%)$ female (mean age $26.6 \pm 1.7$ years $)(P>0.05)$.

The serum TOS was found to be significantly higher in the keratoconus group compared with that of the control group (6.75 \pm 1.07 vs $4.28 \pm 0.34 \mu \mathrm{mol} \mathrm{H}_{2} \mathrm{O}_{2}$ equivalent/l, $P=0.036$ ), whereas serum TAS did not show significant difference between the keratoconus and control groups ( $0.71 \pm 0.05$ vs $0.77 \pm 0.03 \mathrm{mmol}$ Trolox equivalent/l, $P=0.497$ ) (Figures 1 and 2, respectively). Similar to the serum TOS values, the OSI value was significantly higher in the keratoconus group than in the control group (3.08 \pm 1.49 vs $0.61 \pm 0.08$ arbitrary units, $P=0.037)$ (Figure 3).

Comparing the keratoconus severity groups (mild, $n=4$, moderate, $n=9$, severe, $n=12$ ), there were no statistically significant differences in terms of TOS, TAS, and OSI values $(P>0.05)$. Moreover, correlation analysis did not show significant relations between maximum keratometry and oxidative stress markers $(P>0.05)$.

\section{Discussion}

The molecular pathogenesis of keratoconus is poorly understood and the role of oxidative stress is still being investigated. Previous ex vivo studies have demonstrated

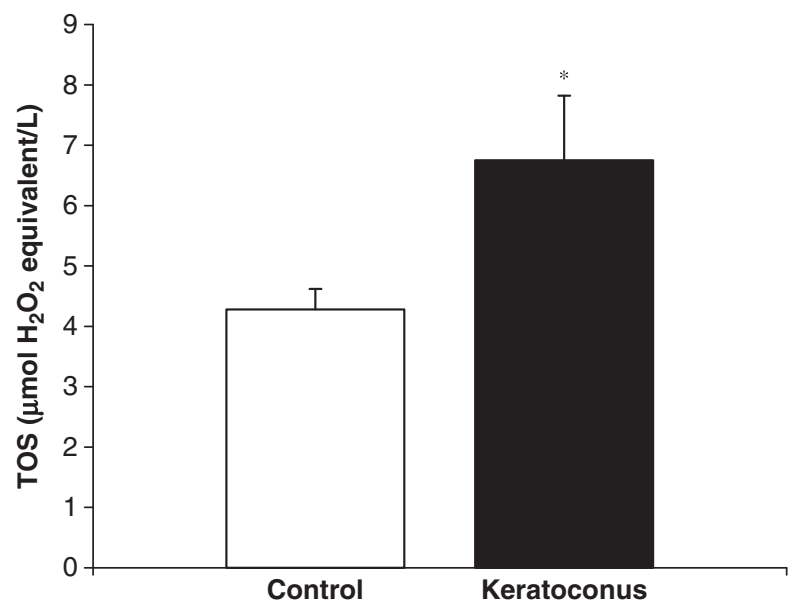

Figure 1 Total oxidant status (TOS) of serum in the control and keratoconus groups. (Values are expressed as mean \pm standard error, ${ }^{*} P=0.036$, Mann-Whitney $U$ test).

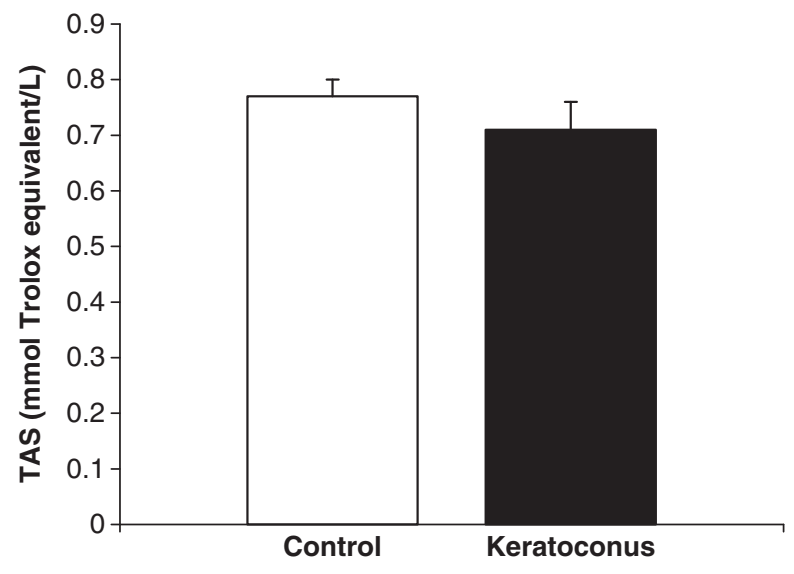

Figure 2 Total antioxidant status (TAS) levels of serum in the control and keratoconus groups. (Values are expressed as mean \pm standard error, $P=0.497$, Mann-Whitney $U$ test).

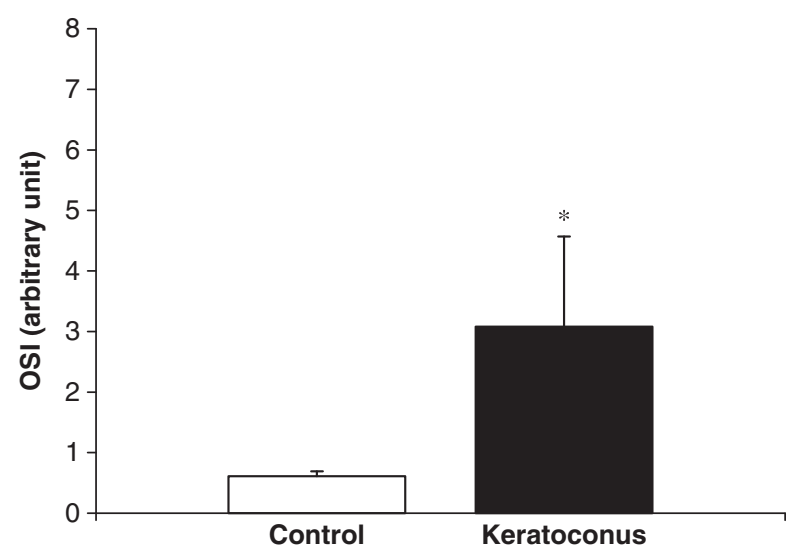

Figure 3 Oxidative stress index (OSI) values for the control and keratoconus groups. (Values are expressed as mean \pm standard error, ${ }^{*} P=0.037$, Mann-Whitney $U$ test). 
higher levels of oxidative byproducts that react with cellular substrates, mitochondrial DNA damage, and altered antioxidant enzymes in keratoconic corneas. ${ }^{14-17}$ Arnal et $a l^{5}$ reported that the total nitrite levels and lipid peroxidation were significantly higher, whereas antioxidant capacity and glutathione content were significantly lower in keratoconic corneas when compared with those of the healthy corneas. Similarly, extracellular superoxide dismutase levels were found to be significantly decreased in keratoconic corneas. ${ }^{8}$ Furthermore, it has been suggested that oxidative stress induces activation of tissue proteinases and degradation of proteinase inhibitors that lead to progressive corneal thinning, a major hallmark of keratoconus ${ }^{9,18}$ Kenney et al ${ }^{19}$ demonstrated reduced levels of tissue inhibitor of matrix metalloproteinase 1 and increased cathepsin V/L2 (a cysteine proteinase) and catalase levels in keratoconic corneas.

Oxidative stress can be defined as an increase in oxidants and/or a decrease in antioxidant capacity. ${ }^{20}$ Although previous ex vivo studies have focused on alterations in oxidant and antioxidant components of keratoconic corneas, systemic oxidative status of the patients with keratoconus was not investigated previously. 4,5,21 To our knowledge, our study is the first to determine oxidant and antioxidant status of the patients with keratoconus by measuring serum TAS and TOS levels.

The measurement of TAS practically represents the cumulative action of antioxidants and their synergistic interaction, which reflects the antioxidant status of the organism. ${ }^{20}$ However, oxidant status and antioxidant status should be measured simultaneously to assess oxidative stress more exactly. ${ }^{20}$ Furthermore, redox balance between oxidation and antioxidation can be determined by the ratio percentage of the TOS to TAS, regarded as OSI. ${ }^{13}$ We used an automated colorimetric measurement method for TAS and TOS developed by Erel, which has been found to be sensitive, reliable, and reproducible. ${ }^{10,12,22}$

In the current study, serum TOS and OSI were found to be significantly higher in patients with keratoconus compared with those of the controls, although there was no significant difference in terms of TAS between the groups. These results might suggest that patients with keratoconus are exposed to potent oxidative stress and redox balance shifts toward oxidation, whereas antioxidant defense seems to be unchanged in these patients. Similarly, a number of clinical studies have found that the tears of patients with keratoconus had lower levels of glutathione (an antioxidant that compensates increased oxidative stress), higher levels of proteolytic activity, and overexpression of several matrix metalloproteinases compared with those of the healthy controls. $^{23,24}$
In conclusion, although previous ex vivo studies have provided evidence that local oxidative stress has a role in the pathogenesis of keratoconus, this is the first clinical study to evaluate the systemic oxidative and antioxidative status of the patients with keratoconus. The higher serum oxidant status and OSI indicate that systemic oxidative stress might contribute to the development of keratoconus, whereas our findings need to be confirmed by further studies in a larger population.

\section{Summary}

\section{What was known before:}

- Previous ex vivo studies demonstrated oxidative damage and alterations in antioxidant defenses in keratoconic corneas. The systemic oxidative status of patients with keratoconus was not investigated previously.

\section{What this study adds:}

- In patients with keratoconus, serum oxidant status and oxidative stress index were significantly higher when compared with those of the healthy controls. This study revealed that systemic oxidative stress might be an important predisposing factor for keratoconus.

\section{Conflict of interest}

The authors declare no conflict of interest.

\section{References}

1 Rabinowitz YS. Keratoconus. Surv Ophthalmol 1998; 42 297-319.

2 Krachmer JH, Feder RS, Belin MW. Keratoconus and related noninflammatory corneal thinning disorders. Surv Ophthalmol 1984; 28: 293-322.

3 Monnies CW. Abnormal rubbing and keractectasia. Eye Contact Lens 2007; 33: 265-271.

4 Buddi R, Lin B, Atilano SR, Zorapapel NC, Kenney MC, Brown DJ. Evidence of oxidative stress in human corneal diseases. J Histochem Cytochem 2002; 50: 341-351.

5 Arnal E, Peris-Martínez C, Menezo JL, Johnsen-Soriano S, Romero FJ. Oxidative stress in keratoconus? Invest Ophthalmol Vis Sci 2011; 52: 8592-8597.

6 Kenney MC, Brown DJ, Rajeev B. Everett Kinsey lecture. The elusive causes of keratoconus: a working hypothesis. CLAO J 2000; 26: 10-13.

7 Shoham A, Hadziahmetovic M, Dunaief JL, Mydlarski MB, Schipper HM. Oxidative stress in diseases of the human cornea. Free Radic Biol Med 2008; 45: 1047-1055.

8 Behndig A, Karlsson K, Johansson BO, Brannstrom T, Marklund SL. Superoxide dismutase isoenzymes in the normal and diseased human cornea. Invest Ophthalmol Vis Sci 2001; 42: 2293-2296.

9 Zhou L, Sawaguchi S, Twining SS, Sugar J, Feder RS, Yue BY. Expression of degradative enzymes and protease inhibitors in corneas with keratoconus. Invest Ophthalmol Vis Sci 1998; 39: 1117-1124.

10 Erel O. A novel automated method to measure total antioxidant response against potent free radical reactions. Clin Biochem 2004; 37: 112-119. 
11 Ferreira SM, Lerner SF, Brunzini R, Evelson PA, Llesuy SF. Oxidative stress markers in aqueous humor of glaucoma patients. Am J Ophthalmol 2004; 137: 62-69.

12 Erel O. A new automated colorimetric method for measuring total oxidant status. Clin Biochem 2005; 38 : 1103-1111.

13 Harma M, Harma M, Erel O. Increased oxidative stress in patients with hydatidiform mole. Swiss Med Wkly 2003; 133 563-566.

14 Atilano SR, Coskun P, Chwa M, Jordan N, Reddy V, Le K et al. Accumulation of mitochondrial DNA damage in keratoconus corneas. Invest Ophthalmol Vis Sci 2005; 46: 1256-1263.

15 Chwa M, Atilano SR, Reddy V, Jordan N, Kim DW, Kenney MC. Increased stress-induced generation of reactive oxygen species and apoptosis in human keratoconus fibroblasts. Invest Ophthalmol Vis Sci 2006; 47: 1902-1910.

16 Chwa M, Atilano SR, Hertzog D, Zheng H, Langberg J, Kim DW et al. Hypersensitive response to oxidative stress in keratoconus corneal fibroblasts. Invest Ophthalmol Vis Sci 2008; 49: 4361-4369.

17 Gondhowiardjo TD, van Haeringen NJ. Corneal aldehyde dehydrogenase, glutathione reductase, and glutathione s-transferase in pathologic corneas. Cornea 1993; 12: 310-314.
18 Smith VA, Easty DL. Matrix metalloproteinase 2: involvement in keratoconus. Eur J Ophthalmol 2000; 10: 215-226.

19 Kenney MC, Chwa M, Atilano SR, Tran A, Carballo M, Saghizadeh $\mathrm{M}$ et al. Increased levels of catalase and cathepsin V/L2 but decreased TIMP-1 in keratoconus corneas: evidence that oxidative stress plays a role in this disorder. Invest Ophthalmol Vis Sci 2005; 46: 823-832.

20 Horoz M, Bolukbas C, Bolukbas FF, Aslan M, Koylu AO, Selek $\mathrm{S}$ et al. Oxidative stress in hepatitis $\mathrm{C}$ infected end-stage renal disease subjects. BMC Infect Dis 2006; 6: 114.

21 Udar N, Atilano SR, Brown DJ, Holguin B, Small K, Nesburn AB et al. SOD1: a candidate gene for keratoconus. Invest Ophthalmol Vis Sci 2006; 47: 3345-3351.

22 Erel O. A novel automated direct measurement method for total antioxidant capacity using a new generation, more stable ABTS radical cation. Clin Biochem 2004; 37: 277-285.

23 Saijyothi AV, Fowjana J, Madhumathi S, Rajeshwari M, Thennarasu M, Prema P et al. Tear fluid small molecular antioxidants profiling shows lowered glutathione in keratoconus. Exp Eye Res 2012; 103: 41-46.

24 Balasubramanian SA, Mohan S, Pye DC, Willcox MD. Proteases, proteolysis and inflammatory molecules in the tears of people with keratoconus. Acta Ophthalmol 2012; 90: e303-e309. 